\title{
Simulation of Wave Interaction with a Circular Ice Floe
}

\author{
Luofeng Huang* \\ Department of Mechanical Engineering \\ University College London \\ London, United Kingdom, WC1E 6BT \\ Email: ucemlhu@ucl.ac.uk \\ Giles Thomas \\ Department of Mechanical Engineering \\ University College London \\ London, United Kingdom, WC1E 6BT \\ Email: giles.thomas@ucl.ac.uk
}

\begin{abstract}
Global warming is inducing sea ice retreat, which is opening new shipping routes and extending the accessible area for resource exploration. This encourages an increasing research interest in sea ice behaviour. With the sea ice melting, level ice is broken up by waves propagated from the open ocean, resulting in an environment where both floating ice floes and waves exist. Such wave-ice interaction can bring significant influences on the potential human activities. This work presents a series of numerical simulations to predict the behaviour of a circular ice floe forced by regular waves, with different wavelength and wave amplitude conditions being investigated. The numerical model was validated against experiments, and it revealed good accuracy in predicting the rigid body motion of an ice floe, including some extreme cases that are difficult to model by previous methods. Two specific behaviours were observed during the numerical simulations, namely overwash and scattering. Both behaviours are discussed in detail to analyse their linear/nonlinear effect on the ice floe motion. The applied model could be used to provide valuable estimations for Arctic engineering purposes.
\end{abstract}

\section{Introduction}

With global warming, the sea ice extent in the Arctic is reducing quickly. Satellite images have observed its summer minimum to have decreased by approximately $12 \%$ per decade [1], Predictions suggest that within the next decades the effects of global warming will transform the Arctic into a seasonally navigable ocean and provide increased impetus for extensive exploration for oil and gas reserves [2].

The effects of ice reduction on human activities in the Arctic can be more complex than anticipated. Rather than providing a pure open ocean environment, the melted ice cover can result in numerous ice floes floating on the sea surface [3]. Such a transition between open ocean and level ice coverage is known as the Marginal Ice Zone (MIZ), in which the extensive human activities are expected to carry out [4]. Thus, research interest has been raising in studying the mechanism of the MIZ and predicting its potential influence on Arctic engineering.

In the MIZ, the waves coming from the open ocean can induce the movement of a solitary ice floe and further lead to the behaviours of multiple ice floes, such as collision [5], herding [6] and rafting [7]. Meanwhile, the incoming waves can be scattered by an ice floe to cause directional spreading or attenuated by a group of ice floes [8]. Under the effect of wave wash and floe-floe collisions, the shape of ice floes in the MIZ tends to be circular, also known as pancake ice, and the diameter of the ice floe is much smaller than the dominating wavelength [9].

Although the ultimate goal of relevant research is to predict the influence of the above processes on maritime activities, to date, only few experimental works have considered the coupling wave-ice effect on a ship $[10,11]$ or a cylinder [12].

${ }^{*}$ Corresponding Author 
Copyright (Frevious studies of the MIZ focussed on the full understanding and accurate modelling of the wave-ice interaction [13, 14]. Based on the long wavelength and small amplitude waves that are common in the MIZ, both experimental and theoretical models usually consider the ocean waves to be regular waves, and the ice floe is assumed to be rigid according to its small dimension.

Meylan et al. [15] conducted wave tank experiments to investigate the wave-induced motions of a solitary floating disk. They recorded the movement trajectory of the disk and decomposed it into six-degrees-of-freedom (6-DOF) motions. Alongside drifting with the wave flow, the disk was found to undergo oscillatory motions at the same frequency as the incident waves. They also observed wave scattering when the dominating wavelength is less than twice of the disk diameter and found the scattering can reduce the motion amplitudes of the disk. However, they used a barrier around the edge of the disk to prevent overwash, i.e. wave running over the top of the disk surface. Based on the findings of Meylan et al. [15], Yiew et al. [16] carried out further experiments to study the influence of overwash on the disk motions, by comparing the motions of two disks, with and without the edge barrier. They found overwash tends to happen with short wavelengths or large wave amplitudes and has a suppression effect on the disk motions.

On account of the high costs in experimental attempts, theoretical models have been developed to provide convenient solutions. Grotmaack and Meylan [17] applied a slope-sliding model to predict the wave-induced surge motion of an ice floe. The model assumes the floe does not affect the incoming wave field, so it is not applicable in a short-wave condition where scattering is expected to happen. Meylan and Squire [18] and Montiel [19] developed a linear potential-flow/thin-plate model that includes the wave field surrounding the floe due to scattering. It can predict the wave-induced surge, heave and pitch motions of an ice floe. After validated against experiments of Yiew et al. [16], this model was shown to be accurate as long as no visible overwash occurring. Overwash has been observed to be a highly non-linear process that can induce an irregular wave field [20], so it is hard to be properly included in a linear model and brings about inaccuracy that may not be neglected. Although Skene et al. [21] incorporated the nonlinear shallow water equations with the linear potential-flow/thin-plate model to predict the depth of the overwash water, their method is based on one-way coupling, i.e. no back coupling from overwash to predict the surrounding fluid domain or the ice floe motions.

Due to the very small freeboard of sea ice, overwash is a highly frequent phenomenon in the Arctic, while a gap remains on accurately modelling it and studying its role in wave-ice interactions. A remedy to this can be using the Computational Fluid Dynamics (CFD) technique to numerically solve the nonlinear Navier-Stokes equations, by which it is possible to obtain a fully-matched solution between the fluid domain and the floating body movement. The wave-ice interaction including overwash is similar to the combination of two common CFD applications: (a) wave response of a floating body (b) green water load. To both of them, related CFD methods have been well applied [22], so technically it is possible to build a CFD model to simulate such a wave-ice interaction. Another reason to apply the CFD method is that it would be easy to use complex geometry files to study the coupling wave-ice effect on offshore structures, such as a ship or a platform.

The above discussion only contains the situation of a solitary ice floe. Although experimental/theatrical works have been conducted on the wave interaction with multiple ice floes, e.g. collision [23] and attenuation [20], the same issue of overwash is also unresolved. Therefore, the current work first focuses on building a valid CFD model to simulate the wave interaction with a solitary ice floe, which will be the foundation to future extended models of multiple ice floes or wave-ice-structure coupling.

In this work, the CFD code Flow-3D [24] was employed to build a three-dimensional model, where a floating circular ice floe is subjected to incident regular waves generated by a numerical wavemaker, as illustrated in Fig. 1. A series of simulations was conducted to capture the surge, heave and pitch motions of the disk in different wavelength and wave amplitude conditions. Validation was conducted against the experiments of Yiew et al. [16] to show the accuracy of the present model and compare it with previous methods. Subsequently, wave overwash and scattering were demonstrated and their effects on the ice floe motions were investigated in detail.

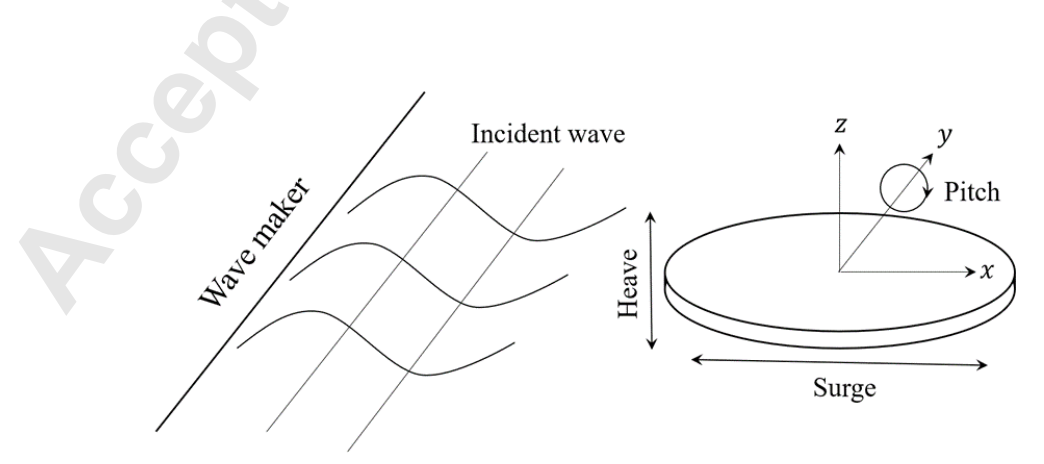

Fig. 1: Schematic of the case: a circular ice floe is freely floating on the water surface and subjected to incoming regular waves generated by a numerical wavemaker, where the surge, heave and pitch motions are its main hydrodynamic responses. 


\subsection{Governing Equations}

The solution of the fluid domain was obtained by solving the Reynolds-averaged Navier-Stokes (RANS) equations for an incompressible Newtonian fluid, as expressed in Eqn. (1) and (2).

$$
\nabla \cdot \overline{\mathbf{v}}=0
$$

$$
\frac{\partial(\rho \overline{\mathbf{v}})}{\partial t}+\nabla \cdot(\rho \overline{\mathbf{v}})=-\nabla \bar{p}+\nabla \cdot\left(\bar{\tau}-\rho \overline{\mathbf{v}^{\prime} \mathbf{v}^{\prime}}\right)+\rho g
$$

where $\overline{\mathbf{v}}$ is the time-average velocity vector $(u, v, w), \mathbf{v}^{\prime}$ is the fluctuating one, $\rho$ stands for the density, $\bar{p}$ denotes the pressure, $\bar{\tau}=\mu\left[\nabla \overline{\mathbf{v}}+(\nabla \overline{\mathbf{v}})^{T}\right]$ is the viscous term, $\mu$ is the dynamic viscosity and $g$ is the gravitational acceleration. As the RANS equations have considered the turbulent fluid, the renormalization-group $k-\varepsilon$ model [25] was adopted to close the equations. The reason to choose this turbulence model is that it is suitable for predicting the complex turbulent and curving flow, which is expected to occur during a complicated wave-ice interaction.

The free surface between the air and water was modelled by the Volume of Fluid (VOF) method [26]. The VOF method introduces a scalar $\alpha$, which denotes the fractional volume of a cell occupied by a specific phase. In this case, a value of $\alpha=1$ corresponds to a cell full of water and a value of $\alpha=0$ indicates a cell full of air. Thus, the free surface, which is a mix of these two phases, is formed by the cells with $0<\alpha<1$. The elevation of the free surface as a function of time is obtained by the advection equation of $\alpha$, as expressed in Eqn. (3). For a cell containing both air and water, its density and viscosity are determined by a linear average of both the fluids, according to Eqn. (4) and (5).

$$
\begin{gathered}
\frac{\partial \alpha}{\partial t}+\nabla \cdot(\overline{\mathbf{v}} \alpha)=0 \\
\rho=\alpha \rho_{\text {water }}+(1-\alpha) \rho_{\text {air }} \\
\mu=\alpha \mu_{\text {water }}+(1-\alpha) \mu_{\text {air }}
\end{gathered}
$$

In this study, $\rho_{\text {water }}=1000 \mathrm{~kg} / \mathrm{m}^{3}, \mu_{\text {water }}=1 \times 10^{-3} \mathrm{~N} \cdot \mathrm{s} / \mathrm{m}^{2} ; \rho_{\text {air }}=1 \mathrm{~kg} / \mathrm{m}^{3}, \mu_{\text {air }}=1.48 \times 10^{-5} \mathrm{~N} \cdot \mathrm{s} / \mathrm{m}^{2}$ and $\mathrm{g}$ was set as $9.81 \mathrm{~m} / \mathrm{s}^{2}$.

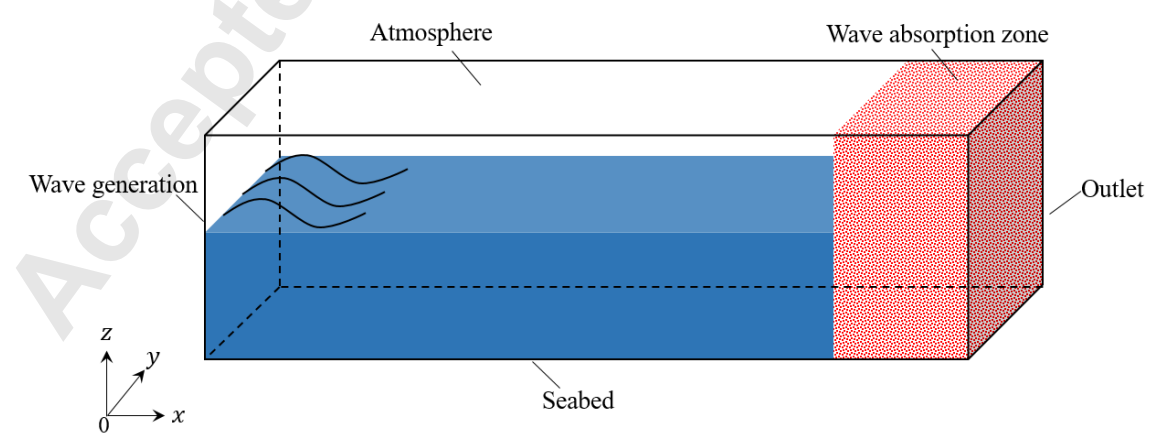

Fig. 2: Sketch of the computational domain and the applied boundary conditions. 


\section{Copyright $6.2^{20}$ Computational Domain and Boundary Conditions}

As shown in Fig. 2, a three-dimensional cuboid computational domain was established, defined by the earth-fixed Cartesian coordinate system $O-x y z$. The $(x, y)$ plane parallels the undistributed water surface, and the $z$-axis is positive upwards. The computational domain is $2 \mathrm{~m}$ wide and $1 \mathrm{~m}$ high, and its length is five times of the target wavelength $(\lambda)$. A no-slip wall condition was applied to the boundary $z=0$ to model the presence of the seabed, and a static pressure condition was defined to the top boundary to account for the atmosphere. The domain was filled with water to a depth of $h=0.83 m$, and the water surface was initialised as still.

The inlet boundary was set at $x=0$, where regular waves are continuously generated and propagating towards the positive $x$-direction, by prescribing the free surface elevation and velocity components according to the linear Stokes wave theory [27]:

$$
\begin{gathered}
\eta=h+\frac{H}{2} \cos (k x-\omega t) \\
u=\frac{\pi H}{T} \frac{\cosh k(z+h)}{\sinh k h} \cos (k x-\omega t) \\
w=\frac{\pi H}{T} \frac{\sinh k(z+h)}{\sinh k h} \sin (k x-\omega t)
\end{gathered}
$$

where $\eta$ is the free surface elevation, $H$ is the wave height, $T$ is the wave period, $k$ is the wave number and $\omega$ is the angular frequency. For the wave properties, $H$ and $T$ are given in advance, and the wavelength $(\lambda=2 \pi / k)$ was solved by the dispersion relation:

$$
k \tanh k h=\kappa, \kappa=\omega^{2} / g
$$

To model an open ocean environment, wave reflections should be avoided at the outlet. This was achieved by placing a wave absorption zone in front of the outlet boundary at the maximal x position. The length of the wave absorption zone is set as one wavelength of the incident wave. Inside the wave absorption zone, an artificial damping term [28] was added to the right hand side of Eqn. (2), evolved into:

$$
\frac{\partial(\rho \overline{\mathbf{v}})}{\partial t}+\nabla \cdot(\rho \overline{\mathbf{v}})=-\nabla \bar{p}+\nabla \cdot\left(\bar{\tau}-\rho \overline{\mathbf{v}^{\prime} \mathbf{v}^{\prime}}\right)+\rho g-\rho \beta\left(\overline{\mathbf{v}}-\overline{\mathbf{v}_{\mathbf{s t r}}}\right)
$$

where $\beta$ is a damping coefficient in units of $s^{-1}$. It linearly increases along wave absorption zone, following Eqn. (11). $\mathbf{v}_{\mathbf{s}}$ is the background stream velocity that is exempted from damping, which was set at 0 since the incident waves are without current speed. Subsequently, the zero-gradient condition was applied to the outlet and other boundaries.

$$
\beta= \begin{cases}\frac{x-\left(x_{\max }-\lambda\right)}{\lambda}, & \text { if } x>x_{\max }-\lambda . \\ 0, & \text { if } x<x_{\max }-\lambda .\end{cases}
$$

\subsection{Ice Modelling and Wave Conditions}

For the purpose of validation, the ice and wave parameters set in this study follow an accordant manner with the experiments of Yiew et al. [16]. As shown in Fig. 3, two rigid disks, with and without an edge barrier, were employed to model ice floes, named as Disk B and Disk NB respectively. The edge barrier attached on Disk B can prevent waves from flowing onto the upper surface of the disk, so that the influences of overwash can be investigated by comparing the wave response of the two disks. The density of the disks was set at $636 \mathrm{~kg} / \mathrm{m}^{3}$.

Each disk was initialised as floating on the undistributed water surface, with its mass centre locating at two wavelengths away from the inlet in the $x$-direction, the middle section of the $y$-direction and its buoyancy-gravity equilibrium position in the $z$-direction. The tested wave conditions cover a wavelength from $\lambda=0.69 \sim 4.91 \mathrm{~m}$ combined with a wave amplitude of $a=10 \sim 40 \mathrm{~mm}$. For each single wave condition, the two disks were put into the CFD model separately. 

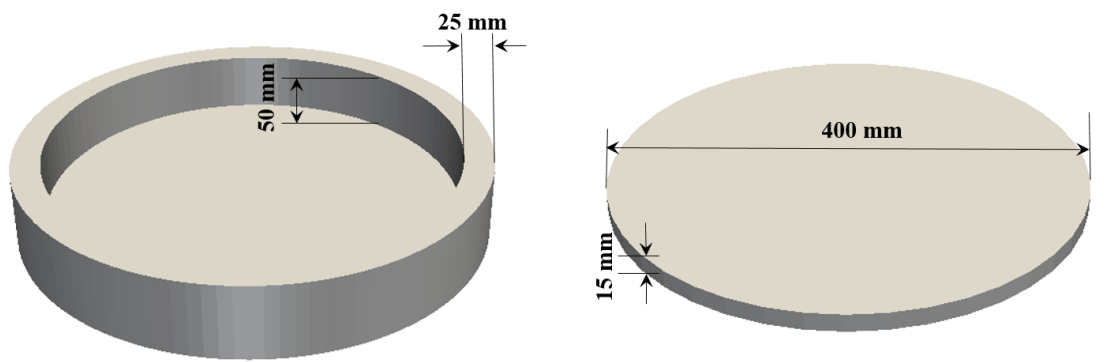

Fig. 3: The geometry of the two disks. Left panel: the disk with an edge barrier (Disk B); right panel: the disk without edge barrier (Disk NB).

\subsection{Ice Floe Motions}

With the generated waves continuously propagating from the inlet to the outlet, the disk is expected to leave its initial equilibrium position and move with the incoming waves. No artificial restraint was applied to the movement, so the disk was allowed to move freely. The wave-induced movement of a disk can be considered as the combination of translation and rotation, which was solved with the rigid-body motion equations in the body-fixed system based on the mass centre of the disk $G-x^{\prime} y^{\prime} x^{\prime}$, as expressed in Eqn. (12) and (13).

$$
\begin{gathered}
\mathbf{F}=m \frac{\mathrm{d} \overrightarrow{V_{G}}}{\mathrm{~d} t} \\
\mathbf{T}=[J] \cdot \frac{\mathrm{d} \overrightarrow{\omega_{G}}}{\mathrm{~d} t}+\overrightarrow{\omega_{G}} \times\left([J] \cdot \overrightarrow{\omega_{G}}\right)
\end{gathered}
$$

Where $\mathbf{F}$ and $\mathbf{T}$ are the total force and torque on the disk, induced by its gravity and the hydraulic load from surrounding fluid $\mathbf{F}_{\mathbf{h}}$, as expressed in Eqn. (14); $m$ and $[J]$ is the mass and inertia moment tensor of the disk, and $\overrightarrow{V_{G}}$ and $\overrightarrow{\omega_{G}}$ are the translational and rotational velocity vectors of the disk.

$$
\mathbf{F}_{\mathbf{h}}=\int(-\bar{P} \mathbf{n}+\bar{\tau} \cdot \mathbf{n}) d S
$$

\subsection{Computational Method}

The Finite Volume Method [29] was applied to obtain the solution of the fluid domain over a certain time duration. The process includes two types of discretisation, in space and time respectively. In space, the computational domain is divided into a set of non-overlapping cells, known as a mesh; in time, the temporal dimension is split into a finite number of timesteps. For a single timestep, the solution of the governing equations can be obtained in each cell (e.g. $\bar{v}, \bar{P}, \alpha)$, and the whole fluid domain can be integrated by the solution of all cells. Then, the fluid domain over a certain time duration is the composition of the fluid domain at each timestep.

In this study, the computational domain was divided into a hexahedral mesh, as shown in Fig. 4. Local mesh refinements were applied at the free-surface area and the area where the disk is expected to move. The cells around the floating disk are partially blocked by the disk volume, and the blockage is described by fractional cell volumes and areas on cell sides [30]. The blockage updates after each timestep to satisfy a two-way fluid-structural coupling.

The size of each timestep was determined by a prescribed value, Courant number $(C)$ :

$$
C=\frac{u \Delta t}{\Delta x}
$$

where $\Delta t$ is the timestep size, $u / \Delta x$ is its normal velocity divided by the distance between the cell centre and the neighbour cell centre. For every timestep, there exists a maximal $u / \Delta x$ value in the domain, and $\Delta t$ can be calculated by the product of that value and $C$. This allows an optimal $\Delta t$ being selected according to the transient fluid state. The value of $C$ was given as 1 in this study. 


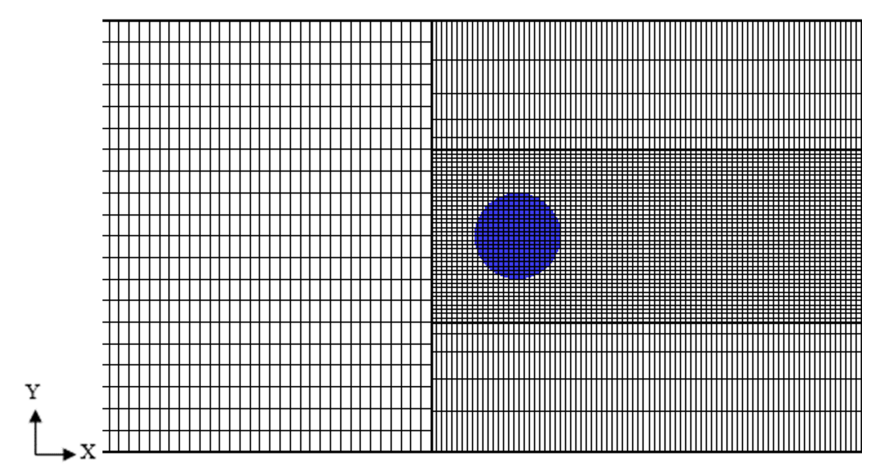

(a) Plan view

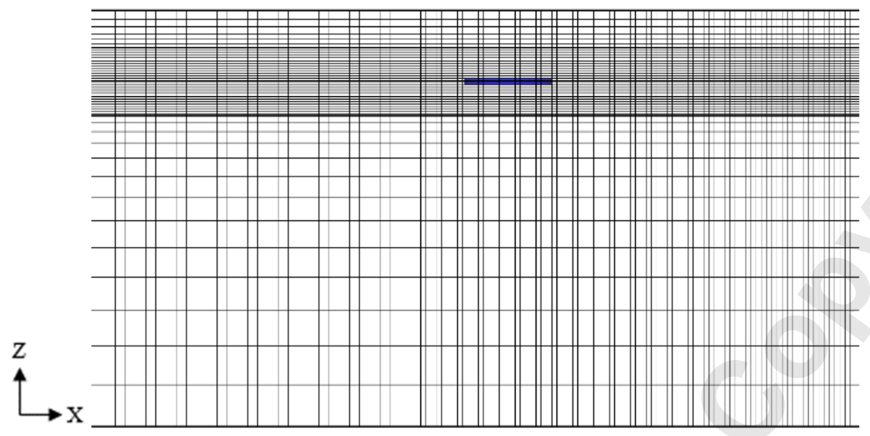

(b) Profile view

Fig. 4: Mesh layout of this CFD model. High resolution was applied to the free surface area and where the disk is expected to move.

\subsection{Data Processing}

With the waves continuously being generated and propagating, a regular wave field gradually forms in the domain, and after that, the disk is expected to conduct periodic motions with stable incoming waves. This indicates the simulation has entered its steady state, and valid data were taken only after the periodic motions begin.

The wave-induced disk movement was decomposed into 6-DOF motions for analysis. As the incident waves are unidirectional, the wave-induced movement of a disk is basically within the $x-z$ plane, composed by translational motions along the $x$-axis and $z$-axis, alongside a rotational motion along the $y^{\prime}$-axis, i.e. surge, heave and pitch. The surge is a combination of a harmonic oscillation with a drift, while the heave and pitch are just harmonic oscillations. These harmonic oscillations were induced by the elliptical wave motion, while the drift was forced by the wave celerity.

To investigate the relationship between the disk motions and incident waves. The surge, heave, pitch amplitudes were calculated and compared with the corresponding wave motion amplitudes through the Response Amplitude Operators (RAOs). After the drift was eliminated, the surge amplitude $\left(a_{s}\right)$ was calculated as half the difference between the average peak and trough values of its oscillation, and the heave amplitude $\left(a_{H}\right)$ and pitch amplitude $\left(a_{P}\right)$ were calculated through the same procedure. Subsequently, the motion amplitudes were used to obtain the RAOs, following:

$$
\begin{gathered}
R A O_{\text {surge }}=\frac{a_{S}}{a \operatorname{coth} k h} \\
R A O_{\text {heave }}=\frac{a_{H}}{a} \\
R A O_{\text {pitch }}=\frac{a_{P}}{k a}
\end{gathered}
$$

\subsection{Mesh Sensitivity Test}

As the computational cost increases with the cell number, mesh sensitivity tests aim to get an accurate solution with as few cells as possible. In this work, two tests were conducted. The first test was to verify the quality of wave generation 
Copyright (c) and $^{2} 18$ propation. For the large wavelength and small amplitude waves that are of interest, at the free surface area the mesh density is sensitive in the vertical direction. Therefore, the cell number per wave height $(M)$ was varied to see the influence on generated waves. For the mesh density in the wavelength direction, 100 cells per wavelength were always used. In the wave tests, the floating disk was taken away and replaced by a probe to record the free surface elevation. Fig. 5 presents the recorded free surface elevation at different $M$ values, alongside the ideal value. Based on the test, $M=15$ was chosen to generate the mesh around the free surface, as the wave field obtained at this density was very close to the target.

The second test was conducted to secure an accurate solution of the disk motions. The mesh density was globally scaled, and four sets of mesh were produced, consisting of 570k, 770k, 970k and $1.25 \mathrm{M}$ cells respectively. The RAOs of the disk were calculated with the four meshes respectively, and the results are shown in Fig. 6. It shows the RAOs converge to stable values with the cell number increased, and the convergent RAO values are close to the experimental data of Yiew et al. [16]. The cell number of 970k was selected, as further increasing the cell number did not effectively improve the results. This set of mesh corresponds to around 40 cells per disk diameter (D) and 6 cells per disk thickness.

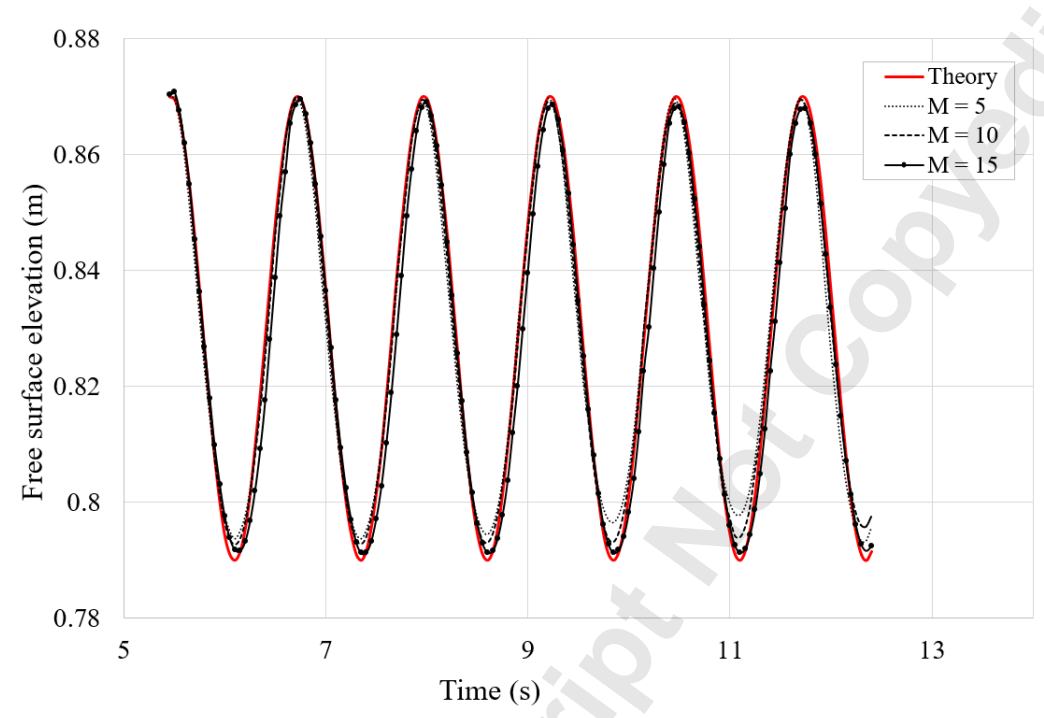

Fig. 5: Generated waves with different cell numbers per wave height. The target waves are of $\lambda=2.38 \mathrm{~m}$ and $a=40 \mathrm{~mm}$

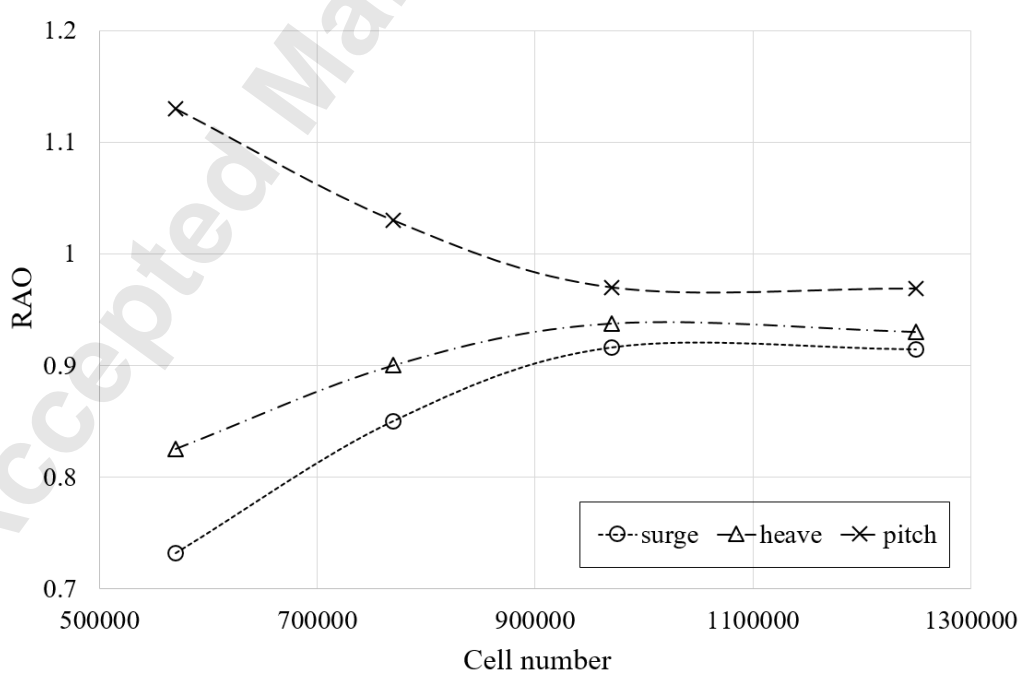

Fig. 6: RAO values with different global cell numbers. The applied wave condition was $\lambda=2.38 \mathrm{~m}$ and $a=40 \mathrm{~mm}$. 


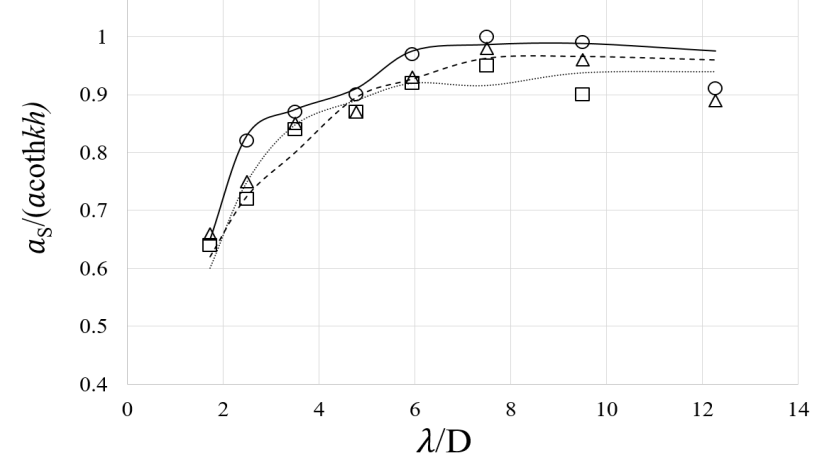

(a) Surge RAO of Disk B

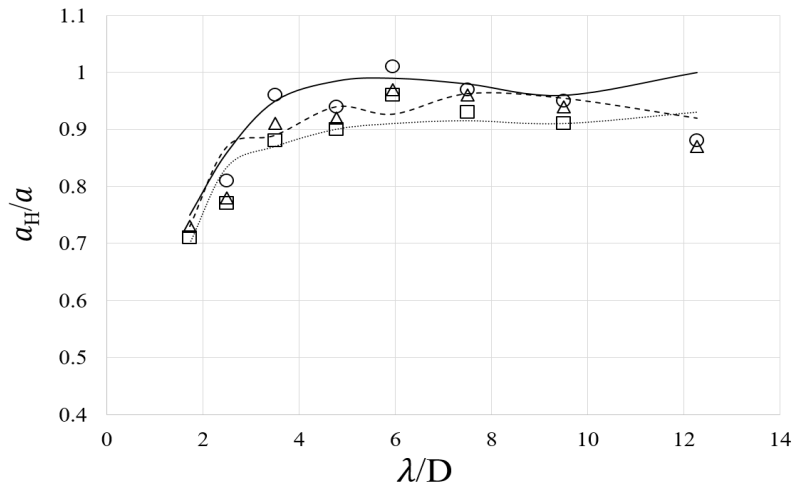

(c) Heave RAO of Disk B

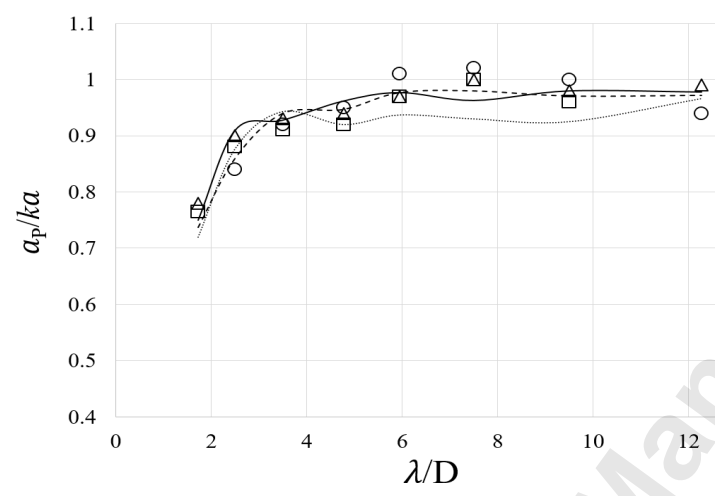

(e) Pitch RAO of Disk B

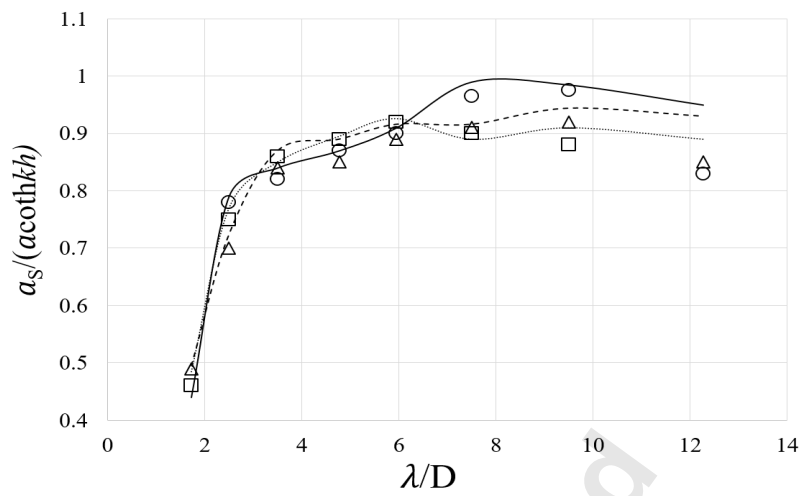

(b) Surge RAO of Disk NB

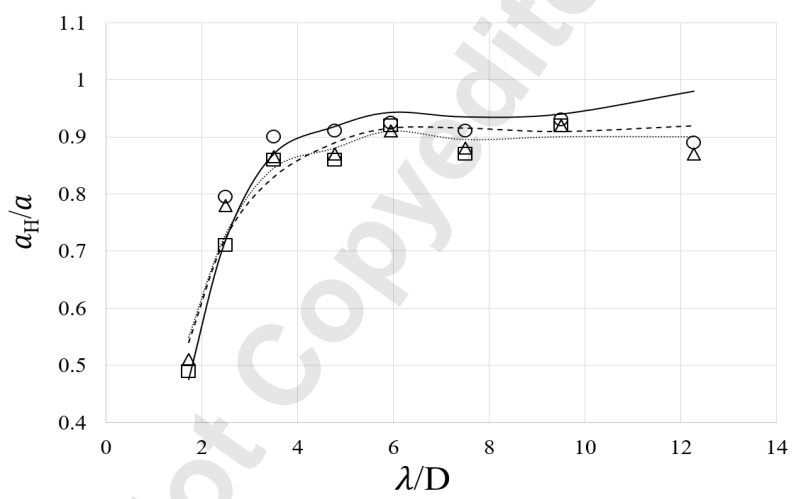

(d) Heave RAO of Disk NB

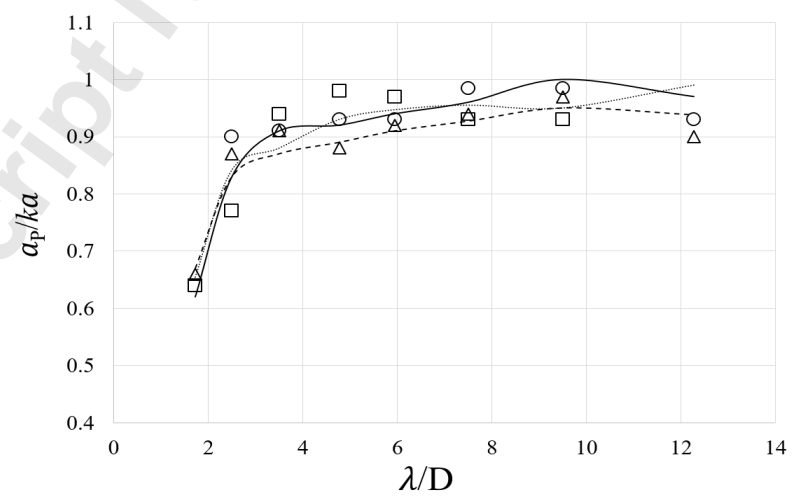

(f) Pitch RAO of Disk NB

\begin{tabular}{|ccc|}
\hline CFD $\mathrm{a}=40 \mathrm{~mm}$ & $\cdots$ CFD $\mathrm{a}=20 \mathrm{~mm}$ & CFD $\mathrm{a}=10 \mathrm{~mm}$ \\
$\circ$ Exp. $\mathrm{a}=40 \mathrm{~mm}$ & $\Delta$ Exp. $\mathrm{a}=20 \mathrm{~mm}$ & $\square$ Exp. $\mathrm{a}=10 \mathrm{~mm}$ \\
\hline
\end{tabular}

Fig. 7: Computational and experimental [16] RAOs, as a function of non-dimensional wavelength.

\section{Results and Discussion}

\subsection{Validation}

The CFD model was validated against the experiments conducted at the University of Tasmania [16]. For the simulations at different wavelength and amplitude conditions, computational results include the surge, heave, pitch RAOs of both Disk $\mathrm{B}$ and Disk NB. The comparison between the computational results (CFD) and experimental data (Exp.) is presented in Fig. 7 , as a function of non-dimensional incident wavelength $(\lambda / D)$. For error analysis, the deviation between the two results was calculated as:

$$
\text { Deviation }=\frac{|C F D-E x p \cdot|}{\operatorname{Exp} .} \times 100 \%
$$




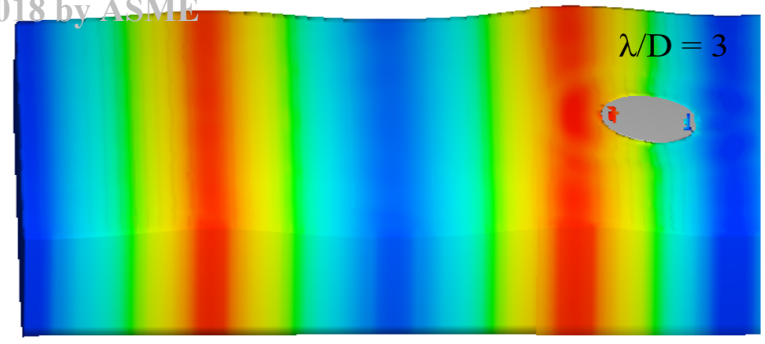

(a) Partial overwash overview
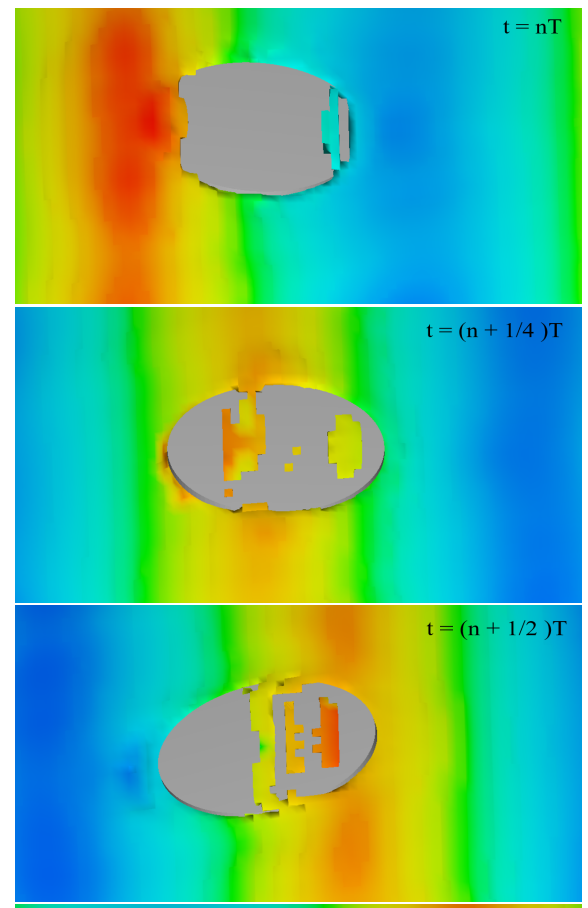

$t=(n+3 / 4) T$
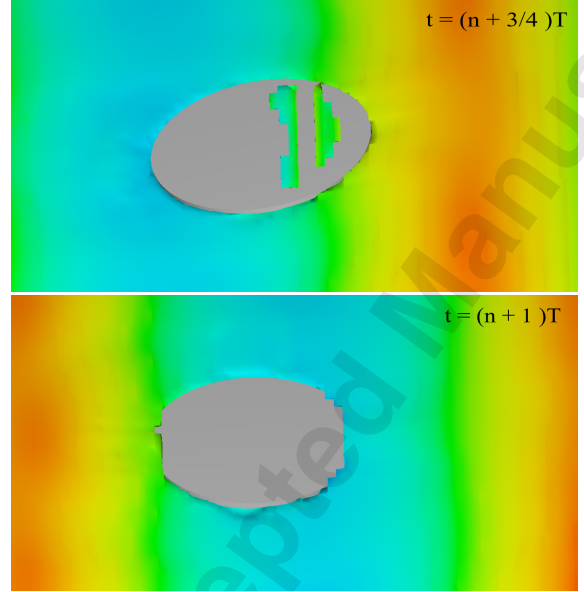

(c) Partial overwash close-up animation

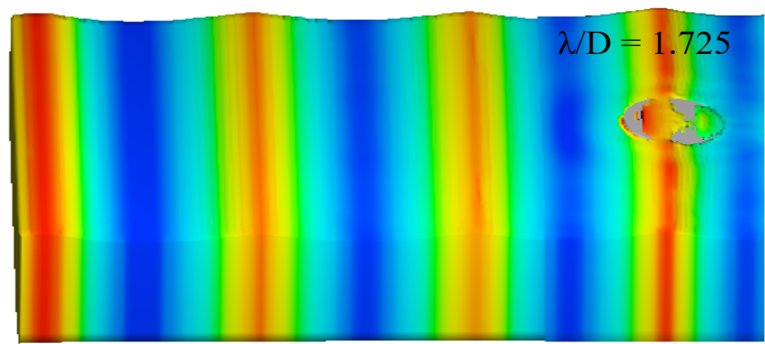

(b) Full overwash overview
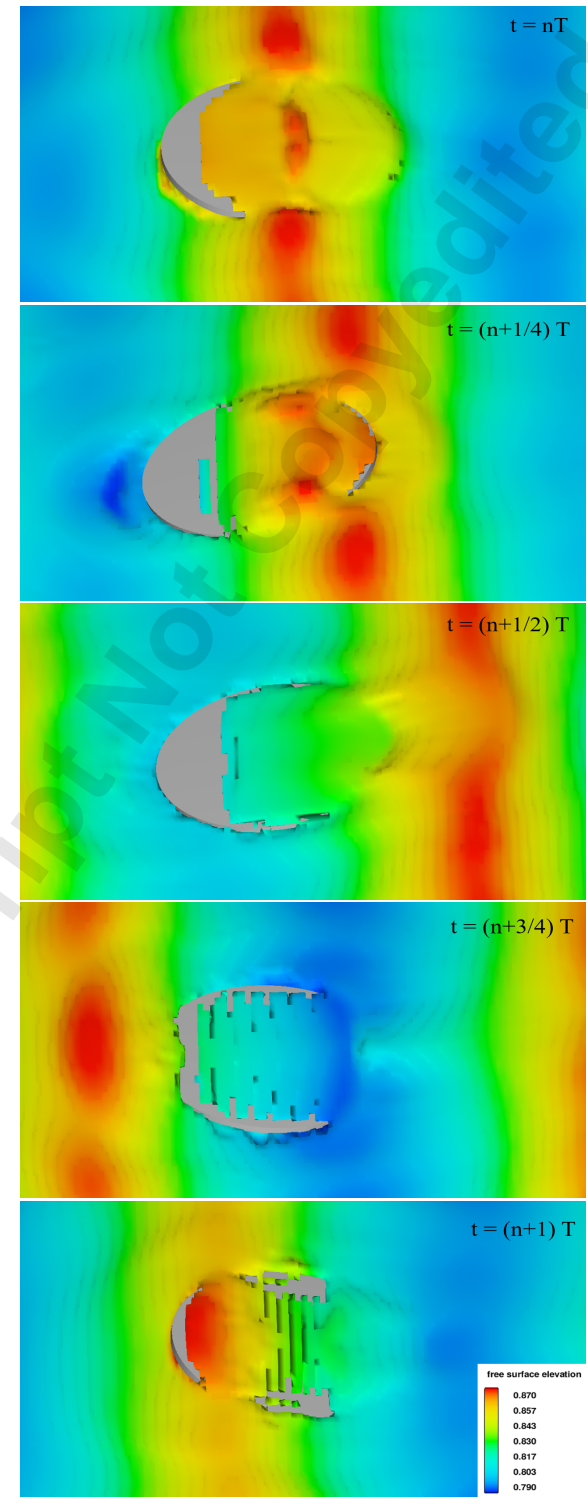

(d) Full overwash close-up animation

Fig. 8: CFD illustration of partial overwash and full overwash.

Overall, the CFD model demonstrates a good agreement with the experiments in predicting the disk motions. For surge, heave and pitch, the mean deviations are $2.4 \%, 2.5 \%$ and $2.8 \%$ respectively. The deviation does not vary significantly at any specific wavelength or amplitude regime. This is because the mesh density of each simulation was set according to the incident wave condition, rather than a constant size. As the deviations are slight and there is no obvious over/underestimate trend identified, the deviations can be considered as the uncertainty of numerical calculation [31] or experimental measurement, which means the applied CFD approach is reasonable and accurate.

The average deviations of Disk B and Disk NB are similar, at 2.7\% and 2.4\% respectively. Previous studies found it 
Copyright (difficult to predict the motions of Disk NB. For example, for the RAOs predicted by the potential-flow/thin-plate model, the average deviations for the three motions are 1\% 4\% for Disk B but increase to $4 \% 7 \%$ for Disk NB [16]. The reason for this difference is the existence of overwash with Disk NB. The study of Bennetts and Williams [20] also indicates that the presence of overwash causes considerable inaccuracy to their prediction models.

Overwash has been found to be a highly nonlinear process [21], so it is unlikely to be included in a linear model. Even for a fully nonlinear CFD model, the method to model the free surface should be carefully chosen. For example, the free surface can also be considered as single-phase by enforcing the free surface boundary conditions on it [32]. This method is not applicable in an overwash situation, where the overwash water can be discontinuous on the floating body [20], so it can no longer be treated as a continuous boundary. Therefore, a two-phase modelling via the VOF method is recommended to handle the overwash problem. Moreover, using an appropriate turbulent model is also of importance, since turbulent flow has been observed when strong overwash happening [21].

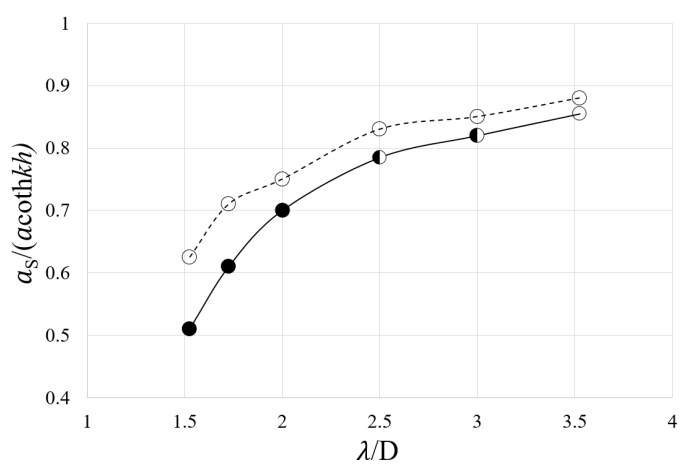

(a) Surge

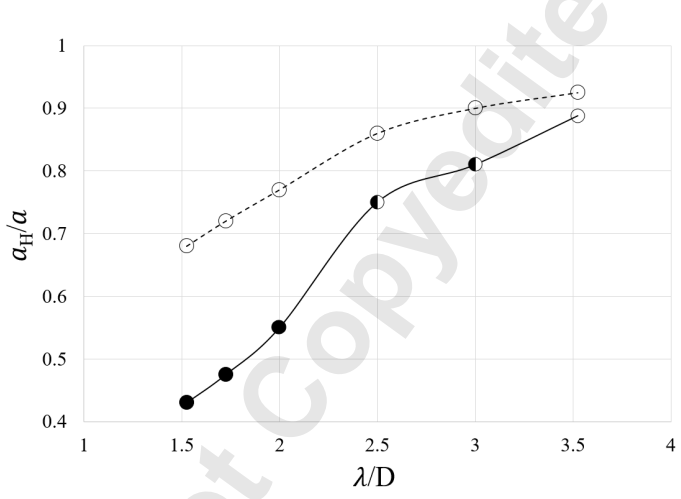

(b) Heave

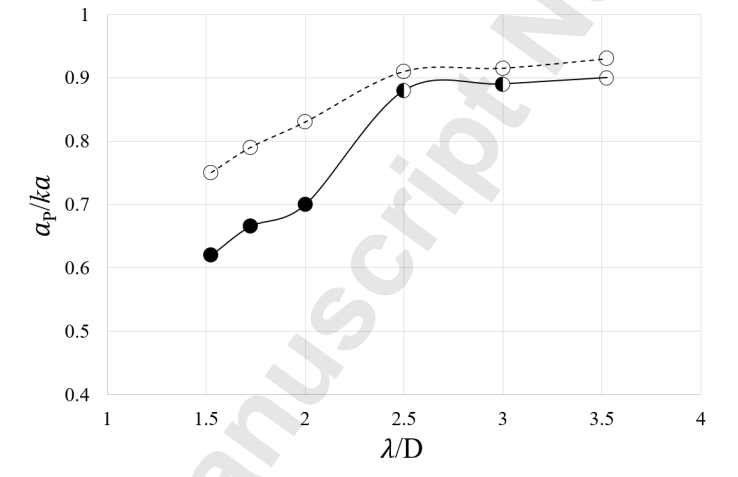

(c) Pitch

○ no overwash $\bullet$ partial overwash • full overwash

Fig. 9: RAO comparisons between Disk B (dash line) and Disk NB (solid line), as a function of non-dimensional wavelength, alongside the type of overwash.

\subsection{Overwash}

Overwash was observed in the simulations of Disk NB. The extent of overwash generally gets stronger with a larger wave amplitude or a smaller wavelength. From weak to strong, two types of overwash were identified, named partial overwash and full overwash. Both are presented in Fig. 8. The process of partial overwash is shown in (a) and (c): water only washes over the head and tail areas of the disk; full overwash is shown in (b) and (d): water flows through the upper surface of the disk.

Since overwash was prevented by the edge barrier of Disk B, the influence of overwash was investigated by comparing the wave response of Disk B and Disk NB. Fig. 9 shows the RAOs of both the disks, together with the observed overwash type. Data were selected from the largest wave amplitude group $(a=40 \mathrm{~mm})$ and in short-wave conditions, where overwash was most obvious. Overwash was inconspicuous in a long-wave condition (when $\lambda / D>3$ ). It can be seen that Disk NB 
Copyright generaliy has smaller RAOs than Disk B. RAO difference between the two disks is most significant for the heave and least for the surge. For surge and heave, the RAO difference increases with a stronger overwash. This results from the load of the overwash water on top of the disk, which suppresses the transitional movement of the disk. Pitch seems to be unaffected by partial overwash, which means the water at head and tail areas of the disk may provide rotational torque, acting as an offset to the extra water load. In contrast, full overwash can suppress pitch notably.

The influence of wave amplitude on overwash is presented in Fig. 10, where only heave RAO is presented since surge and pitch reveal similar trends. It shows the RAO of Disk B is insensitive to wave amplitude. For Disk NB, its RAO is insensitive to wave amplitude when partial overwash occurs. However, when full overwash appears, the RAO decreases obviously as wave amplitude increases. This is because a larger wave amplitude increases the water depth above the disk, which can enhance the suppressing effect. The fact that the RAOs vary with wave amplitude signifies the nonlinearity of overwash. In the linear theory, the RAOs should be constant values for a single wavelength, but the result indicates such a theory is not applicable when notable overwash occurs.

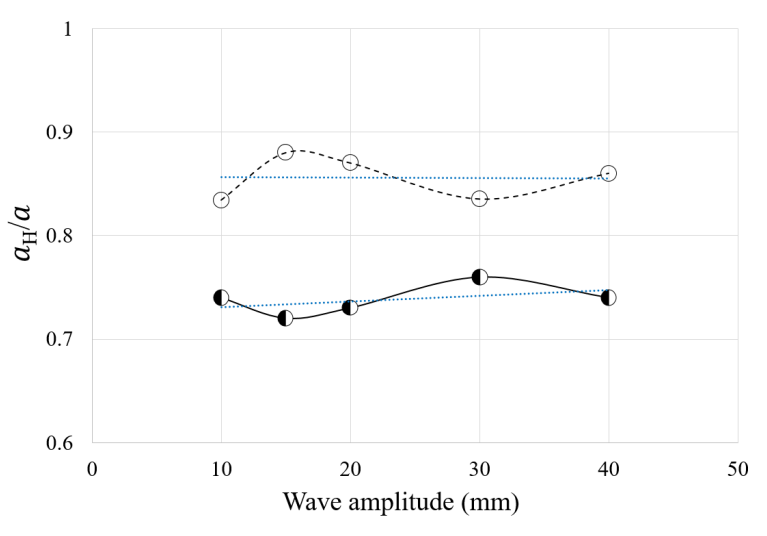

(a) $\lambda / D=2.5$

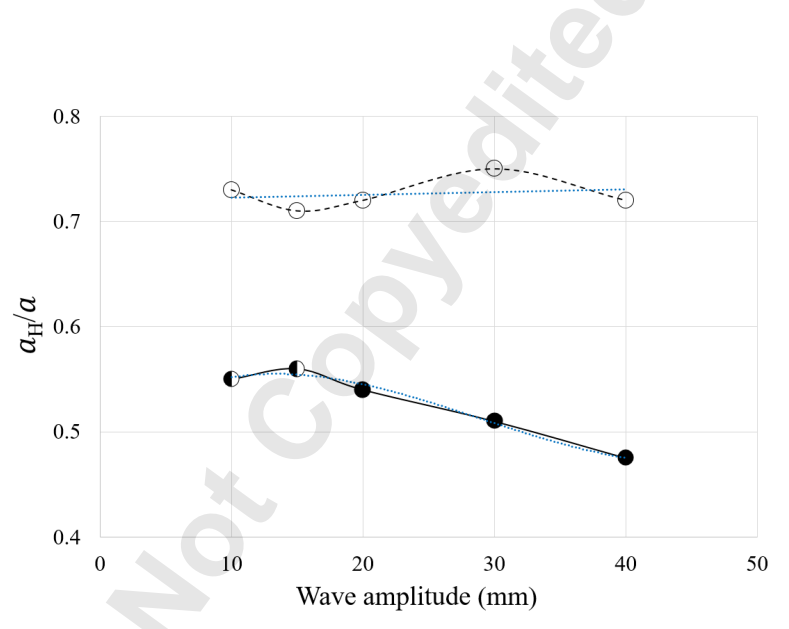

(b) $\lambda / D=1.725$

○ no overwash $\bullet$ partial overwash • full overwash

Fig. 10: Heave RAO of Disk B (dash line) and Disk NB (solid line), as a function of wave amplitude, alongside the type of overwash. Trend lines (dot line) are also included.

\subsection{Wave Scattering}

The disks were observed to reveal varying wave-ice interactions with the incident wavelength changed. When a disk is subjected to long waves, it hardly influences the wave transmission. By contrast, in short waves, a disk can scatter the incoming waves, which generates directional-spreading waves surrounding the disk and influences the transited waves. Examples are shown in Fig. 11, where Disk B is presented, in order to eliminate the distraction of overwash. In a long-wave condition, wave scattering is not obvious, and the waves are nearly intact after passing through the disk, as shown in Fig. 11 (a). At a shorter wavelength, wave scattering becomes visible, while the waves can still transit with the incident amplitude, except the part right behind the disk is distorted, as shown in Fig. 11 (b). For even shorter waves, a stronger scattering can be observed, and the transited waves are significantly attenuated, as shown in Fig. 11 (c).

The disk motions are related to the scattering phenomenon. As shown in Fig. 7, in the long-wave regime $(\lambda / D>3)$, wave scattering is negligible and the RAOs are close to one, which means the disk approximately moves at the same amplitude of the incident waves, agreeing with the fact that the wave transmission is unaffected. In the short-wave regime $(\lambda / D<3)$, the RAOs start to decrease rapidly with the scattering becomes stronger, since the scattering can attenuate the incident waves so that the disk motions cannot be induced at the original wave amplitude.

Although both scattering and overwash have been found as sources to deform the incident wave and can reduce the disk RAOs, they reveal different relationships with the incident wave amplitude. As shown in Fig. 10, for Disk B, where only scattering occurs, the RAO is insensitive with a changed incident wave amplitude, which suggests a linear effect of scattering on the disk motions. However, for Disk NB, overwash has shown obvious nonlinearity on the disk motions. This explains why the linear potential-flow/thin-plate model can predict the RAOs accurately in a scattering condition as long as there is no overwash. 


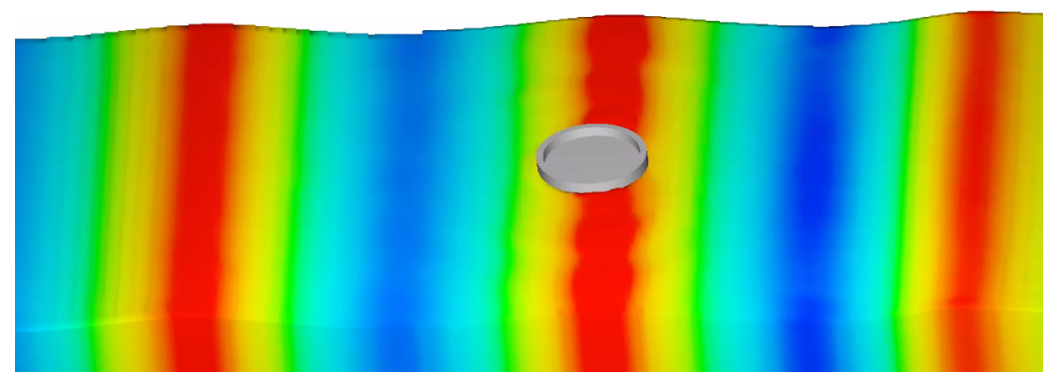

(a) $\lambda / D=3.525$

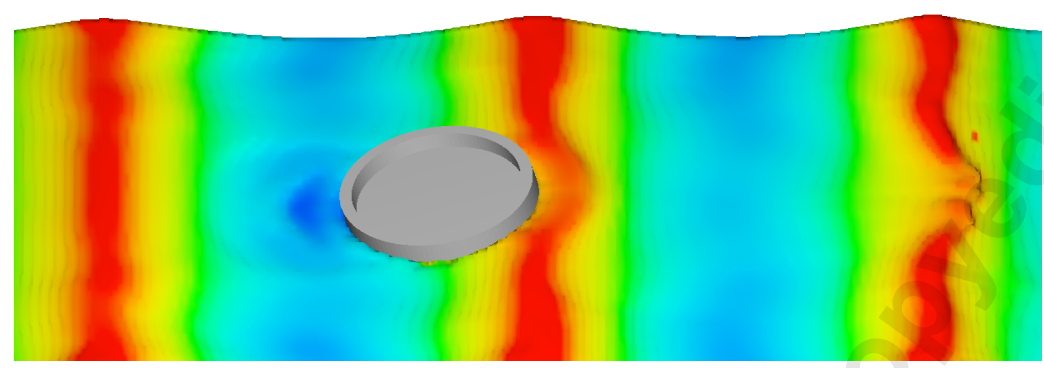

(b) $\lambda / D=2$

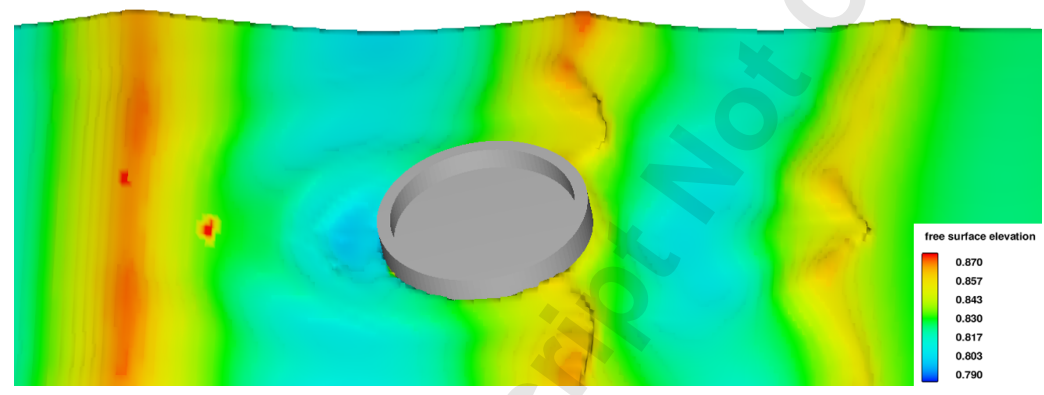

(c) $\lambda / D=1.525$

Fig. 11: The interaction of Disk B with waves, at different wavelengths.

\section{Conclusions}

A CFD model has been introduced to model the interaction of regular waves with a circular ice floe. The ice floe was treated as a floating rigid disk, and it was subjected to waves of large wavelength and small amplitude, according to the common environment in the MIZ. Relevant numerical theories and approaches have been presented in detail.

Based on Flow-3D, a series of simulations have been conducted to investigate the behaviour of an ice floe in different wave conditions. The wave-induced surge, heave and pitch motions of an ice floe were presented as RAOs to analyse their relationship with incident waves. The computational results were compared with the corresponding experimental data. A small level of deviation exists in each simulation, which can be considered reasonable and signifies the used approach is valid. The presented CFD approach reveals pleasing capability to simulate the wave-ice interaction including overwash, to which previous models have been reported to be inaccurate.

Two specific behaviours with the wave-ice interaction were displayed within the simulations, namely overwash and scattering. Both tend to happen in a short-wave condition and were found to be significant sources to cause wave dissipation and reduce the ice floe motions. The scattering and ice floe motions were found to follow a linear relationship that is not affected by a changed the wave amplitude, but such a relationship becomes nonlinear when overwash occurs, where a larger wave amplitude can increase the water depth on top of an ice floe, resulting in extra load to suppress the motions.

This work provided a valid CFD model to simulate nonlinear wave-ice interactions. Although the simulations presented are in model-scale for purpose of validation, in principle there is no reason that the approach cannot be applied at fullscale. Moreover, the CFD model can be easily extended to involve multiple ice floes or be incorporated with fixed/floating structures, which could be considered in future work. 


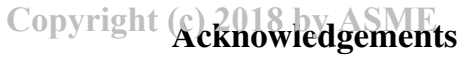

Thanks go to Prof G.X. Wu at University College London (UCL), who provided insightful suggestions to improve this paper. The first author is grateful to Lloyds Register Foundation, UCL Faculty of Engineering Science and China Scholarship Council, for funding his PhD study. This work is part of a project that has received funding from the European Union's Horizon 2020 research and innovation programme under grant agreement No 723526 - SEDNA: Safe maritime operations under extreme conditions; the Arctic case.

\section{References}

[1] Stroeve, J. C., Kattsov, V., Barrett, A., Serreze, M., Pavlova, T., Holland, M., and Meier, W. N., 2012. "Trends in arctic sea ice extent from cmip5, cmip3 and observations". Geophysical Research Letters, 39(16).

[2] Smith, L. C., and Stephenson, S. R., 2013. "New trans-arctic shipping routes navigable by midcentury". Proceedings of the National Academy of Sciences, 110(13), pp. E1191-E1195.

[3] Thomson, J., Squire, V., Ackley, S., Rogers, E., Babanin, A., Guest, P., Maksym, T., Wadhams, P., Stammerjohn, S., and Fairall, C., 2013. Sea state and boundary layer physics of the emerging arctic ocean. Tech. rep., WASHINGTON UNIV SEATTLE APPLIED PHYSICS LAB.

[4] Lee, C. M., Cole, S., Doble, M., Freitag, L., Hwang, P., Jayne, S., Jeffries, M., Krishfield, R., Maksym, T., and Maslowski, W., 2012. Marginal ice zone (miz) program: Science and experiment plan. Tech. rep., WASHINGTON UNIV SEATTLE APPLIED PHYSICS LAB.

[5] Martin, S., and Becker, P., 1987. "High-frequency ice floe collisions in the greenland sea during the 1984 marginal ice zone experiment". Journal of Geophysical Research: Oceans, 92(C7), pp. 7071-7084.

[6] Wadhams, P., 1983. "A mechanism for the formation of ice edge bands". Journal of Geophysical Research: Oceans, 88(C5), pp. 2813-2818.

[7] Dai, M., Shen, H. H., Hopkins, M. A., and Ackley, S. F., 2004. "Wave rafting and the equilibrium pancake ice cover thickness". Journal of Geophysical Research: Oceans, 109(C7).

[8] Montiel, F., Squire, V., and Bennetts, L., 2016. "Attenuation and directional spreading of ocean wave spectra in the marginal ice zone". Journal of Fluid Mechanics, 790, pp. 492-522.

[9] Thomson, J., Ackley, S., Girard-Ardhuin, F., Ardhuin, F., Babanin, A., Boutin, G., Brozena, J., Cheng, S., Collins, C., Doble, M., Fairall, C., Guest, P., Gebhardt, C., Gemmrich, J., Graber, H. C., Holt, B., Lehner, S., Lund, B., Meylan, M. H., Maksym, T., Montiel, F., Perrie, W., Persson, O., Rainville, L., Rogers, W. E., Shen, H., Shen, H., Squire, V., Stammerjohn, S., Stopa, J., Smith, M. M., Sutherland, P., and Wadhams, P., 2018. "Overview of the arctic sea state and boundary layer physics program". Journal of Geophysical Research: Oceans.

[10] Luo, W.-Z., Guo, C.-Y., Wu, T.-C., and Su, Y.-M., 2018. "Experimental research on resistance and motion attitude variation of ship-wave-ice interaction in marginal ice zones". Marine Structures, 58, pp. 399-415.

[11] Guo, C.-y., Xie, C., Zhang, J.-z., Wang, S., and Zhao, D.-g., 2018. "Experimental investigation of the resistance performance and heave and pitch motions of ice-going container ship under pack ice conditions". China Ocean Engineering, 32(2), pp. 169-178.

[12] McGovern, D. J., and Bai, W., 2014. "Experimental study of wave-driven impact of sea ice floes on a circular cylinder". Cold Regions Science and Technology, 108, pp. 36-48.

[13] Squire, V. A., Dugan, J. P., Wadhams, P., Rottier, P. J., and Liu, A. K., 1995. "Of ocean waves and sea ice”. Annual Review of Fluid Mechanics, 27(1), pp. 115-168.

[14] Squire, V., 2007. "Of ocean waves and sea-ice revisited”. Cold Regions Science and Technology, 49(2), pp. $110-133$.

[15] Meylan, M. H., Yiew, L. J., Bennetts, L. G., French, B. J., and Thomas, G. A., 2015. "Surge motion of an ice floe in waves: comparison of a theoretical and an experimental model”. Annals of Glaciology, 56(69), pp. $155-159$.

[16] Yiew, L., Bennetts, L., Meylan, M., French, B., and Thomas, G., 2016. "Hydrodynamic responses of a thin floating disk to regular waves". Ocean Modelling, 97, pp. 52-64.

[17] Grotmaack, R., and Meylan, M. H., 2006. "Wave forcing of small floating bodies". Journal of waterway, port, coastal, and ocean engineering, 132(3), pp. 192-198.

[18] Meylan, M. H., and Squire, V. A., 1996. "Response of a circular ice floe to ocean waves". Journal of Geophysical Research: Oceans, 101(C4), pp. 8869-8884.

[19] Montiel, F. F., 2012. "Numerical and experimental analysis of water wave scattering by floating elastic plates". PhD thesis, University of Otago.

[20] Bennetts, L., and Williams, T., 2015. "Water wave transmission by an array of floating discs". In Proceedings of the Royal Society of London A: Mathematical, Physical and Engineering Sciences, Vol. 471, The Royal Society, p. 20140698.

[21] Skene, D., Bennetts, L., Meylan, M., and Toffoli, A., 2015. "Modelling water wave overwash of a thin floating plate". Journal of Fluid Mechanics, 777. 
Copyright $\{22]^{20}$ Jasak, H., 2017 . “Cfd analysis in subsea and marine technology”. In IOP Conference Series: Materials Science and Engineering, Vol. 276, IOP Publishing, p. 012009.

[23] Yiew, L., Bennetts, L., Meylan, M., Thomas, G., and French, B., 2017. "Wave-induced collisions of thin floating disks". Physics of Fluids, 29(12), p. 127102.

[24] Flow Science Inc, 2015. Flow-3d users manual, v11.1.

[25] Yakhot, V., and Orszag, S. A., 1986. "Renormalization group analysis of turbulence. i. basic theory". Journal of scientific computing, 1(1), pp. 3-51.

[26] Hirt, C. W., and Nichols, B. D., 1981. "Volume of fluid (vof) method for the dynamics of free boundaries". Journal of computational physics, 39(1), pp. 201-225.

[27] Dean, R. G., and Dalrymple, R. A., 1991. Water wave mechanics for engineers and scientists, Vol. 2. World Scientific Publishing Company.

[28] Wei, G., 2015. "The sponge layer method in flow-3d".

[29] Versteeg, H. K., and Malalasekera, W., 2007. An introduction to computational fluid dynamics: the finite volume method. Pearson Education.

[30] Bhinder, M. A., Mingham, C. G., Causon, D. M., Rahmati, M. T., Aggidis, G. A., and Chaplin, R. V., 2009. "A joint numerical and experimental study of a surging point absorbing wave energy converter (wraspa)". In ASME 2009 28th International Conference on Ocean, Offshore and Arctic Engineering, American Society of Mechanical Engineers, pp. 869-875.

[31] Freitas, C. J., 2002. “The issue of numerical uncertainty”. Applied Mathematical Modelling, 26(2), pp. 237-248.

[32] Tuković, Ž., and Jasak, H., 2012. "A moving mesh finite volume interface tracking method for surface tension dominated interfacial fluid flow”. Computers \& fluids, 55, pp. 70-84. 